\title{
GEOMETRY WITHIN A LINEAR SPHERICAL COMPLEX*
}

\author{
BY \\ PERCEY F. SMITH
}

Introduction.

The following pages are devoted to the study of a point-sphere correspondence of involutory character, which appears as direct generalization from a certain point of view of the well known point-sphere correspondence arising in a dilatation and the point-point correspondence of spherical inversion. Problems resulting from the establishment by this correspondence of a spherical contact transformation are discussed.

Elsewhere in the Transactions $\dagger$ I have shown the importance to the higher spherical geometry of the involutory contact-transformation here studied, for its relation to the group of all spherical contact transformations is precisely that of spherical inversion to the group of the geometry of reciprocal radii.

The titles of the sections indicate to a great extent their contents.

\section{§1. Point-sphere correspondence: definition and discussion.}

The oquatio directrix in the rectangular point coördinates $x, y, z, X, Y, Z$,

$$
\begin{aligned}
\Phi \equiv\left(X^{2}\right. & \left.+Y^{2}+Z^{2}-r_{0}^{2}\right)\left(x^{2}+y^{2}+z^{2}-r_{0}^{2}\right) \\
& +r_{0}^{2}\left(1-\kappa_{0}^{2}\right)\left[(X-x)^{2}+(Y-y)^{2}+(Z-z)^{2}\right]=0,
\end{aligned}
$$

establishes in space $R_{3}$ the most general involutory point-sphere correspondence $\Gamma$ such that the $\infty^{3}$ coaxial systems determined each by a point and its associated sphere intersect in a fundamental sphere $\sigma$, viz., $x^{2}+y^{2}+z^{2}=r_{0}^{2}$. Under (1) the point $P(x, y, z)$ corresponds to the sphere $S$ of center $x^{\prime}, y^{\prime}, z^{\prime}$ and radius $r^{\prime}$ where

$$
\frac{x^{\prime}}{x}=\frac{y^{\prime}}{y}=\frac{z^{\prime}}{z}=\frac{r_{0}^{2}\left(1-\kappa_{0}^{2}\right)}{x^{2}+y^{2}+z^{2}-r_{0}^{2} \kappa_{0}^{2}},
$$

\footnotetext{
* Presented to the American Mathematical Society October 27, 1900. Received for publication February 23, 1901.

† On surfaces enveloped by spheres belonging to a linear spherical complex, vol. 1 (1900), p. 371.
} 


$$
r^{\prime}=\frac{r_{0} \kappa_{0}\left(x^{2}+y^{2}+z^{2}-r_{0}^{2}\right)}{x^{2}+y^{2}+z^{2}-r_{0}^{2} \kappa_{0}^{2}}
$$

The spheres $S$ in question are subjected to a single condition. The elimination of $x, y, z$ from (2) and (3) gives

$$
{x^{\prime}}^{2}+{y^{\prime}}^{2}+{z^{\prime}}^{2}-r_{0}^{2}-{r^{\prime}}^{2}-2 r_{0} r^{\prime} \kappa=0,
$$

if

$$
\kappa=\frac{1+\kappa_{0}^{2}}{2 \kappa_{0}} \text {. }
$$

The $\infty^{3}$ spheres $S$, therefore, belong to a linear spherical complex $A$ whose fundamental sphere is $\sigma$ and whose "constant" equals $\kappa$.

Special features of $\Gamma$ are the following :-Points on $\sigma$ correspond to themselves. Points on the sphere $\sigma_{0}\left(0,0,0,-r_{0} \kappa_{0}\right)$ give the $\infty^{2}$ planes of $A$, and these all touch the sphere $\sigma_{0}^{\prime}\left(0,0,0,-r_{0} \kappa\right)$. The sphere $\sigma_{0}$ belongs to $A$. $A n y$ point of $R_{3}$ at infinity and not on the imaginary circle $C$ corresponds to $\sigma_{0}$, while a point $P$ of $C$ gives the $\infty^{1}$ spheres of radius $-r_{0} \kappa_{0}$ whose centers lie upon the line joining $P$ with the origin. Therefore the fundamental configurations of $\Gamma$ are the points of $C$ in $R_{3}$ and the sphere $\sigma_{0}$ of $A$.

A property of the correspondence of prime importance is established by the equation

$$
\begin{aligned}
\left(x_{1}^{\prime}-x_{2}^{\prime}\right)^{2}+\left(y_{1}^{\prime}-y_{2}^{\prime}\right)^{2}+\left(z_{1}^{\prime}-z_{2}^{\prime}\right)^{2}-\left(r_{1}^{\prime}-r_{2}^{\prime}\right)^{2} & \\
& =\frac{r_{0}^{2}\left(1-\kappa_{0}^{2}\right)\left[\left(x_{1}-x_{2}\right)^{2}+\left(y_{1}-y_{2}\right)^{2}+\left(z_{1}-z_{2}\right)^{2}\right]}{\left(x_{1}^{2}+y_{1}^{2}+z_{1}^{2}-r_{0}^{2} \kappa_{0}^{2}\right)\left(x_{2}^{2}+y_{2}^{2}+z_{2}^{2}-r_{0}^{2} \kappa_{0}^{2}\right)},
\end{aligned}
$$

in which $S_{1}^{\prime}\left(x_{1}^{\prime}, y_{1}^{\prime}, z_{1}^{\prime}, r_{1}^{\prime}\right)$ and $S_{2}\left(x_{2}^{\prime}, y_{2}^{\prime}, z_{2}^{\prime}, r_{2}^{\prime}\right)$ are taken as corresponding to $P_{1}\left(x_{1}, y_{1}, z_{1}\right), P_{2}\left(x_{2}, y_{2}, z_{2}\right)$, respectively. This gives

Theorem 1. The $\infty^{1}$ points $P$ of a minimum line intersecting $\sigma$ in $P_{0}$ become, under $\Gamma, \infty^{1}$ spheres of $A$ touching one another at $P_{0}$.

By considering the minimum cone with vertex $P$, we easily get a second result:

$A$ sphere $S$ of a linear spherical complex $A$ is touched by $\infty^{2}$ spheres of $A$ along its circle of intersection with the fundamental sphere. $\dagger$

This circle we call the trajectory circle of $S$.

Let $E$ be any plane passing through $P$, and let the two minimum lines drawn in $E$ through $P$ intersect $\sigma$ in $p_{1}, p_{2}$. Then $p_{1}, p_{2}$ are vertices of a skew quadrilateral $p_{1} p_{2} P_{1} P_{2}$ of minimum lines lying in $S$, while $p_{1} p_{2}, P_{1} P_{2}$ are polar lines with respect to $S$, since the planes $p_{1} P_{1} p_{2}, p_{1} P_{2} p_{2}$ touch $S$ at $P_{1}$ and $P_{2}$ respectively. Denoting these planes by $E_{1}, E_{2}$, we have the result:

* Cf. Transactions, p. 383. For $\kappa_{0}=0$, the correspondence is the usual inversion in $\sigma$. Evidently the case $\kappa_{0}^{2}=1$ is of no interest.

†Lik, Mathematische Annalen, vol. 5, p. 207. 
Theorem 2. A surface element $(P, E)$ corresponds under $\Gamma$ to two elements $\left(P_{1}, E_{1}\right),\left(P_{2}, E_{2}\right)$ of the sphere $S . \quad$ The planes $E, E_{1}, E_{2}$ intersect in the radical plane of $S$ and $\sigma$, while the circle drawn through $P, P_{1}, P_{2}$ is orthogonal to $E, S, \sigma$.

For example, if $P$ is at infinity in $E$ and not on the imaginary circle, then $\left(P_{1}, E_{1}\right)$ and $\left(P_{2}, E_{2}\right)$ are on $\sigma_{0}$ while $E_{1}, E_{2}, E$ are parallel.

If, now, $P$ moves along the minimum line $P p_{1}$, then $P_{1}$ and $P_{2}$ are displaced on $p_{1} P_{1}$ and $p_{1} P_{2}$ respectively, this being true for any plane $E$ through $P p_{1}$. Therefore,

$A$ minimum line regarded as defined by its $\infty^{2}$ surface elements becones under $\Gamma$ two minimum lines whose plane belongs to $A$, the three lines intersecting on $\sigma$.

The result just stated is of fundamental importance, for we may at once deduce the following properties:

A sphere $s$ becomes under $\Gamma$ two spheres $s_{1}$ and $s_{2}$, and $s_{1}, s_{2}, s$ intersect on $\sigma$. If $s$ degenerates into a plane, then $s_{1}$ and $s_{2}$ touch the sphere $\sigma_{0}$.

The spheres determined by $P_{1} p_{1} p_{2} P$ and $P_{2} p_{1} p_{2} P$ are invariant. Hence the planes tangent to either along its intersection with $\sigma$ must be invariant, i. e., must touch the sphere $\sigma_{0}$. The conclusion is :

TheORem 3. The spheres of a linear spherical complex $A_{0}$ with fundamental sphere $\sigma$ and constant $\kappa_{0}$ are invariant under $\Gamma$. The $\infty^{2}$ spheres of $A_{0}$ tangent to a given sphere $s$ also touch each of two spheres $s_{1}$ and $s_{2}$, into which s goes over by $\Gamma$.

From the properties enunciated in this last theorem, I have called the transformation of space which $\Gamma$ establishes inversion in the complex $A_{0},{ }^{*}$ and have symbolized it by $\left(A_{0}\right)$.

Theorem 2 may be given another interesting form. For if we denote the two spherical images of the plane $E$ on the sphere $S$ by $p_{0}$ and $p_{0}^{\prime}$, i. e., the line joining $p_{0}$ and $p_{0}^{\prime}$ is that diameter of $S$ which is perpendicular to $E$, then the spherical inversion in the center $P$ which leaves $S$ invariant transforms $p_{0}$ and $p_{0}^{\prime}$ into $P_{1}$ and $P_{2}$ respectively. Thus we have the result:

By the spherical inversion with center $P$ which transforms $S$ into itself, the spherical inage on $S$ of any surface element $(P, E)$ inverts into the point $P_{1}$ of the corresponding surface element $\left(P_{1}, E_{1}\right)$.

Next consider all surface elements with common $P$ whose planes intersect in a line $L$. The special representation of these elements on $S$ gives the points of the great circle $C$ whose axis is parallel to $L$. Then the transformed elements lie along the small circle $c$ on $S$ into which $C$ inverts in the center $P$. The circle $c$ intersects the trajectory circle of $S$ orthogonally, since its plane passes through the pole of the radical plane of $P$ and $S$ with respect to $S$.

* Cf. Transactions, vol. 1, p. 376. 
The results established in this section hold with certain obvious exceptions for any number of dimensions, although the reasoning here given has been restricted to the case of ordinary space.

\section{§2. The corresponding contact transformation.}

We proceed now to find the equations of $\left(A_{0}\right)$ as a contact transformation.

For convenience, we write in (1) $x_{1}, x_{2}, x_{3}$ for $x, y, z$, and similarly for $X, Y, Z$. As coördinates of the surface element $(P, E)$ we may take

$$
x_{\mathrm{i}}, x_{2}, x_{3} ; p_{1}, p_{2}, p_{3}, \text { or }(x, p)
$$

if $p_{1}: p_{2}: p_{3}$ determine the direction of the normal to $E$. Let $(P, E)$ become $(X, P)$ by $\left(A_{0}\right)$; then we have to determine $X$ and $P$ from the equations $\dagger$

$$
\Phi=0, \quad \rho p_{i}-\frac{\partial \Phi}{\partial x_{i}}=0, \quad \rho P_{i}+\frac{\partial \Phi}{\partial X_{i}}=0 \quad(i=1,2,3),
$$

after elimination of $\rho$.

The result is

$(6)^{*}$

$$
\{
$$

$$
\begin{aligned}
& X_{i}=x_{i}-\frac{\left(x_{i} s-p_{i} \delta\right)}{s\left(u+\delta^{2}\right)-2 \delta l}\left(u-r_{0}^{2}\right) \\
& P_{i}=\frac{p_{i}\left(u-\delta^{2}\right)-2 x_{i}(l-\delta s)}{r_{0}^{2}-\delta^{2}}
\end{aligned}
$$$$
(i=1,2,3) \text {, }
$$

where

$$
u=\sum_{i} x_{i}^{2}, \quad s^{2}=\sum_{i} p_{i}^{2}, \quad l=\sum_{i} p_{i} x_{i}, \quad \delta=-r_{0} k_{v} .
$$

To these may be added, if $S^{2}=\sum_{i} P_{i}^{2}$,

$$
S=\frac{s\left(u+\delta^{2}\right)-2 \delta l}{r_{0}^{2}-\delta^{2}}
$$

The equation $s\left(u+\delta^{2}\right)-2 \delta l=0$ defines the congruence of spheres of radius $\delta$ and with centers on the minimum cone whose vertex is the center of $\sigma$.

The element $(P, E)$ is invariant when, and only when, $P$ is on $\sigma\left(u=r_{0}^{2}\right)$ and $E$ is tangent to $\sigma_{0}(l-\delta s=0)$. This result shows that the spheres of the complex $A_{0}$ are unchanged by $\left(A_{0}\right)$ as already stated.

The following special results are to be noted, and may be readily verified.

An element $(P, E)$ such that $P$ is on $\sigma$ and $E$ belongs to $A$ transforms into $\infty^{1}\left(P^{\prime}, E^{\prime}\right)$ 's, where $E^{\prime}$ is a minimum plane, and $P^{\prime}$ any point on the minimum line in $E^{\prime}$ through $P$.

An element $(P, E)$ belonging to $\sigma_{0}$ gives the $\infty^{1}$ elements of $E$ at infinity.

*Cf. Lie-EngeL, Trunsformationsgruppen, vol. II, p. 151.

† Transactions, loc. cit., p. 385. As remarked above, $\delta==0$ gives inversion in $\sigma$. 
An element $(P, E)$ of the imaginary circle at infinity goes over into the $\infty^{2}$ elements of the sphere into which $P$ inverts under $\left(A_{0}\right)(\S 1)$.

\section{§3. Duality of the geometry of reciprocal radii and geometry within a linear spherical complex.}

The group of the geometry within a linear spherical complex $A$ is that subgroup $G_{10}$ of the general $G_{15}$ of all sphere-sphere contact transformations under which $A$ is invariant. Any transformation of $G_{10}$ is compounded of inversions in complexes in involution with $A$. The contact transformation $\left(A_{0}\right)$ which carries $A$ over into the complex of all points transforms $G_{10}$ into the group of the geometry of reciprocal radii, every transformation of which is compounded. of spherical inversions. Thus within the general higher spherical geometry, the two geometries mentioned appear equally justified. This result is fundamental for what follows. Let us now assign to the infinite region the characteristics demanded by the geometry of reciprocal radii. Then the points on the imaginary circle are to be excluded and the infinite region becomes the "point at infinity." The fundamental configurations of the correspondence $\Gamma(\S 1)$ disappear, while for $\left(A_{0}\right)$ the $\infty^{2}(P, E)$ 's whose points are on $\sigma$ and whose planes touch $\sigma_{0}^{\prime}$ constitute the fundamental configuration. These go over into the $\infty^{3}$ minimum elements (finite).

We may now mention a number of the more interesting correspondences in the two geometries; in each case the first statement refers to the geometry of reciprocal radii.

Surface $f$, point locus.

Surface $F$, enveloped by $\infty^{2}$ spheres of $A$.

Curve of intersection $c$ of $f$ and $\sigma$. Double curve $c$ of $F$ on $\sigma$.

Curve of intersection $c^{\prime}$ of $f$ and $\sigma_{0}$. Developable of bitangent planes to $F$, enveloping $\sigma_{0}^{\prime}$.

Principal spheres of $f$.

Principal spheres of $F$.

Minimum developable intersecting $\sigma$ Curve $c_{0}$. in $c_{0}$.

Singular line of curvature on $f$, viz., the curve of contact of $f$ with the circumscribing minimum developable $f^{\prime}$.

Line of curvature on $F$ lying on $\sigma$, along which the principal spheres have four-point contact; this line of curvature and the double curve $c$ above mentioned constitute the entire intersection of $F$ and $\sigma .^{*}$

\footnotetext{
* This singular line of curvature on any surface $f$ is determined by differentiation and elimination. These operations suffice also for the determination of the line of curvature in question on $F$.
} 
Confocal surfaces, i. e., surfaces in- Surfaces enveloped by $\infty^{2}$ spheres of scribed in a minimum developable $f^{\prime}$. $A$ and inscribed in a developable, whose planes belong to $A$, along a line of curvature on $\sigma$.

Lines of curvature on $f$. Lines of curvature on $F$.

Minimum lines on $f$ : Minimum lines on $F$.

The congruence of $\infty^{2}$ circles orthogonal at once to $f$ and the fundamental sphere $\sigma$ intersect $F$ also orthogonally, such that each circle cuts $F$ in the two points of contact of an enveloping sphere ( $(3)$. The $\infty^{1}$ circles of this congruence intersecting $f$ along any curve trace out upon $F$ the corresponding curve.

It will be remarked that the developables whose planes belong to $A$, i. e., touch the sphere $\sigma_{0}^{\prime}$, play an analogous rôle in the geometry here discussed to that of minimum developables in the geometry of reciprocal radii. The first mentioned surfaces are remarkable for the fact that their lines of curvature (non-linear) lie upon spheres concentric with the fundamental sphere.

Continuing the discussion of the duality, we have as corresponding :

Curve $C$, point locus.

Point of intersection of $C$ and $\sigma$.

Points of intersection of $C$ and $\sigma_{0}$.

Minimum developable passed through

$C$ and intersecting $\sigma$ in $c$.
Surface $F$ enveloped by $\infty^{1}$ spheres of $A$, i. e., an annular surface.

Nodes on $F$.

Planes touching $F$ along circles.

Line of curvature $c$ on $F$, along which the principal spheres have four-point contact, the complete insection of $F$ and $\sigma$.

Minimum curve, cuspidal edge of mini- Curve $c$.

mum developable intersecting $\sigma$ in $c$.

The locus of centers $\left(x^{\prime}, y^{\prime}, z^{\prime}\right)$ of the spheres of $A$ enveloping $F$ is found from $f$ by the point transformation defined by equations $(2), \S 1$.*

The conformal property of transformations of the group of the geometry of reciprocal radii is expressed in the geometry of surface elements as follows :$(P, E)$ and $\left(P, E^{\prime}\right)$ go over into $\left(P^{\prime}, E_{1}\right)$ and $\left(P^{\prime}, E_{1}^{\prime}\right)$ such that the angle of $E E^{\prime}$ equals that of $E_{1} E_{1}^{\prime}$. For definiteness orientation must be assigned to the planes in question. Now by inversion in the complex $\left(A_{0}\right)$ which transforms $P$ into $S,(P, E)$ and $\left(P, E^{\prime}\right)$ go over into the elements of $S,\left(P_{1} E_{1}\right)$ and $\left(P_{2}, E_{2}\right)$, and $P_{1}$ and $P_{2}$ lie on a circle of $S$ orthogonal to the trajectory circle

\footnotetext{
* For a discussion of the locus of centers, see Transactions, vol. 1, p. 383, seq.
} 
$(\S 1)$, while the minimum elements of the pencil $(P, E),\left(P, E^{\prime}\right)$, invert into the elements common to these two circles. We have then from the results of the last part of $\S 1$ the

Theorem. If the linear spherical complex $A$ invert by $\left(A_{0}\right)$ into the complex of all points so that $(P, E),\left(P, E^{\prime}\right)$ become $\left(P_{1}, E_{1}\right),\left(P_{1}^{\prime}, E_{1}^{\prime}\right)$ on the sphere $S$, and if the circle on $S$ through $P_{1}$ and $P_{1}^{\prime}$ orthogonal to the trajectory circle intersect the latter in $P_{0}$ and $P_{0}^{\prime}$, then the logarithm of the cross ratio $\left(P_{1} P_{0}, P_{1}^{\prime} P_{0}^{\prime}\right)$ divided by $2 \sqrt{ }-1$ equals one-half the angle of the planes $E$ and $E^{\prime}$, and this cross ratio is unaltered by every transformation of $G_{10}$.

Let, now, a curve $C$ invert in $\left(A_{0}\right)$ into the annular surface $F$ enveloped by $\infty^{1}$ spheres of $A$. Any curvature strip* along $C$ becomes under $\left(A_{0}\right)$ a curvature strip on $F$, say along the curve $c$ on $F$. By the theorem of JoAchimsTHAL, any strip along $C$ intersecting that mentioned under a constant angle is also a curvature strip, therefore inverts under $\left(A_{0}\right)$ into a curvature strip on $F$, and in this way all non-circular lines of curvatures $c$ on $F$ are found. Hence the result:

Theorem of Joachimsthal. Two curvature strips along a curve intersect under a constant angle.
The non-circular lines of curvature of an annular surface whose spheres belong to a linear spherical complex determine projective ranges on the circles of curvature. $\dagger$

In the same way we get as dual theorems :

Theorem of Dupin. Any system of $\infty^{3}$ surfaces mutually orthogonal intersect along lines of curvature.
If in any system of $\infty^{3}$ surfaces enveloped by the spheres of a linear spherical complex $A$ the six points of contact of every sphere of the complex are the intersections of three mutually orthogonal circles on that sphere whose common orthogonal circle is the trajectory circle, then the annular surface enveloped by $\infty^{1}$ spheres of $A$ and tangent to a surface of the system along a line of curvature will touch also a second surface of the system along a line of curvature.

\footnotetext{
* i. e., a system of $\infty^{1}$ elements belonging to $C$ whose normals form a developable surface.

$\dagger$ This theorem holds for all annular surfaces. But when the enveloping spheres belong to a linear spherical complex the non-circular lines of curvature are determined by quadratures, for a particular solution of the corresponding RICCATI equation is known, viz., the line of curvature $c$ above mentioned.
} 
Other theorems of interest might be added to those already given, but enough has been given to justify the study of the duality.

It is evident that the general surface within a linear spherical complex will intersect the plane at infinity only along the imaginary circle. Furthermore, such a surface will contain no finite minimum element, whence the result:

$A$ generul algebraic surface in the geometry within a general linear spherical complex is of equal order and class, cutting the plane at infinity only in the imaginary circle, and the enveloping minimum developable is singular.

\$4. The group of inversions in all linear spherical complexes with common fundamental sphere.

If $(A)$ and $\left(A^{\prime}\right)$ are inversions in complexes $A$ and $A^{\prime}$ having a common fundamental sphere $\sigma$, then *

$$
\left(A A^{\prime}\right) \equiv\left(\Omega A^{\prime \prime}\right),
$$

where $(\Omega)$ denotes change in orientation and $A^{\prime \prime}$ also has $\sigma$ for fundamental sphere. If $\kappa, \kappa^{\prime}, \kappa^{\prime \prime}$ are the constants of $A, A^{\prime}, A^{\prime \prime}$ respectively, then

$$
\kappa^{\prime \prime}= \pm \frac{1-\kappa \kappa^{\prime}}{\kappa-\kappa^{\prime}} \text {. }
$$

Now $(\Omega)$ results from $(6)$ and $(7)$ when $\delta=\infty$, for then $(x ; p)$ becomes $(x ; p)$ but $s$ changes sign. Hence $(\Omega)$ plays here the rôle of the identical transformation, and we have

TheOREM 4. The equations (6) define a group $G_{1}$ of contact transformations in the parameter $\kappa_{0}\left(\delta=-r_{0} \kappa_{0}\right)$. The composition law is given by (8).

We find the infinitesimal transformation $U(f)$ of $G_{1}$ in the usual way by setting $\delta=\infty+\delta t$, and obtain

$$
\frac{\delta x_{i}}{\delta t}=\frac{p_{i}}{s}\left(u-r_{0}^{2}\right), \quad \frac{\delta p_{i}}{\delta t}=-2 x_{i} s \quad(i=1,2,3) ;
$$

therefore $G_{1}$ is generated by

$$
\begin{aligned}
U(f) \equiv \frac{u-r_{0}^{2}}{s}\left(p_{1} \frac{\partial f}{\partial x_{1}}+p_{2} \frac{\partial f}{\partial x_{2}}+\right. & \left.p_{3} \frac{\partial f}{\partial x_{3}}\right) \\
& -2 s\left(x_{1} \frac{\partial f}{\partial p_{1}}+x_{2} \frac{\partial f}{\partial p_{2}}+x_{3} \frac{\partial f}{\partial p_{3}}\right) .
\end{aligned}
$$

We determine now the invariants of $G_{1}$. The equation $U(f)=0$ has the six solutions

* This follows from Theorem 4, p. 378, Transactions, vol. 1 (1900). 


$$
\left\{\begin{array}{rr}
a_{3} \equiv x_{1} p_{2}-x_{2} p_{1}, \quad a_{1} \equiv x_{2} p_{3}-x_{3} p_{2}, \quad a_{2} \equiv x_{3} p_{1}-x_{1} p_{3}, \\
\beta_{i} \equiv x_{i} l-\frac{1}{2} p_{i}\left(u+r_{0}^{2}\right) & (i=1,2,3),
\end{array}\right.
$$

connected by the identical relation

$$
a_{1} \beta_{1}+a_{2} \beta_{2}+a_{3} \beta_{3} \equiv 0,
$$

but otherwise independent. Hence

TheOREM 5. Every function invariant under $G_{1}$ has the form

$$
F\left(a_{1}, a_{2}, a_{3}, \beta_{1}, \beta_{2}, \beta_{3}\right),
$$

where $F$ is homogeneous of dimension zero.

The $a, \beta$ have an interesting geometrical significance. For, if $L$ is the line of intersection of the plane $E$ of $(P, E)[(x ; p)]$ with the radical plane of $P$ and the fundamental sphere $\sigma$, then the equations of $L$ in current coördinates $X_{1}, X_{2}, X_{3}$ are

$$
a_{3} X_{1}=a_{1} X_{3}+\beta_{2}, \quad a_{3} X_{2}=a_{2} X_{3}+\beta_{1},
$$

i. e., the $a, \beta$ are the line coördinates of $L$. The plane through $P$ perpendicular to $L$ is

$$
a_{1} X_{1}+a_{2} X_{2}+a_{3} X_{3}=0
$$

and if $C$ be its intersection with $L$, then the coördinates of $C$ are

and

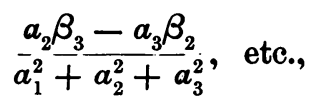

These results give

$$
\overline{C P}^{2}=\frac{\sum_{i} \beta_{i}^{2}}{\sum_{i} a_{i}^{2}}-r_{0}^{2}
$$

THEOREM 6. By the transformations of $G_{1}$ upon any surface element $(P, E)$ the point $P$ is made to describe the circle orthogonal at once to $E$ and the fundamental sphere, while E turns round the axis of this circle.

For the coördinates of the axis, the center, and the radius of the circle in question are invariants.

The most general system of $\infty^{4}$ elements invariant under $G_{1}$ will be defined by an equation of the form

$$
F\left(a_{1}, a_{2}, a_{3}, \beta_{1}, \beta_{2}, \beta_{3}\right)=0,
$$

$F$ being homogeneous.

This equation, however, also defines a line complex, the locus of $\infty^{3}$ axes $(a ; \beta)$ of the circles of Theorem 5 . We are therefore led to a solution of the problem :-To determine all partial differential equations of the first order 
the integral surfaces of which cut orthogonally a given system of $\infty^{3}$ circles orthogonal to a given sphere $\sigma$.

For the given system of circles is uniquely defined by the line complex formed of their axes, $(a, \beta)$ i. e., by an equation (11). If then we replace the $a$ and $\beta$ from equations $(10)$ the problem is solved.*

The " characteristic function" of the infinitesimal homogeneous contact transformation $U(f)$ is

since $\dagger$

$$
\begin{gathered}
H \equiv s\left(u-r_{0}^{2}\right), \\
U(f) \equiv \sum_{i=1}^{3}\left(\frac{\partial H}{\partial p_{i}} \frac{\partial f}{\partial x_{i}}-\frac{\partial H}{\partial x_{i}} \frac{\partial f}{\partial p_{i}}\right) .
\end{gathered}
$$

From theorem 5 we see that the $\infty^{1}$ surfaces resulting from transformation of an arbitrary surface by the group $G_{1}$ belong, by the theorem of RIBaUcour, to an orthogonal system. Furthermore, under $U(f)$ the lines of curvature go over into lines of curvature. Finally, SopHUs LIE has found that the most general infinitesimal contact transformation possessing these properties $\ddagger$ has for characteristic function

$$
H \equiv s\left(a_{0} u+a_{1} x_{1}+a_{2} x_{2}+a_{3} x_{3}+a_{4}\right),
$$

into which $U(f)$ transforms by a translation.

§5. Generalization to space of $n$ dimensions; in particular, to $n=2$.

The preceding discussion may readily be generalized to $n$ dimensions. Theorems 1-5 hold, with trivial changes, in general.

Confining ourselves for brevity to the discussion of the problem mentioned in $\S 4$ for the case $n=2$, we may state the result :

'The most general differential equation of the first order whose integral curves cut orthogonally a given system of $\infty^{1}$ circles orthogonal to a given circle. $\sigma$ is determined thus :-

The center of $\sigma$ being the origin and its radius $r_{0}$, let the given system of circles be defined by the locus of centers

$$
F(a, \beta, \gamma)=0,
$$

$F$ being homogeneous in the homogeneous rectangular coördinates $a, \beta, \gamma$. Then setting

* Writing, of course,

$$
p=\frac{\partial x_{3}}{\partial x_{1}}=-\frac{p_{1}}{p_{3}}, \quad q=\frac{\partial x_{3}}{\partial x_{2}}=-\frac{p_{2}}{p_{3}}
$$

The integration problem of such a differential equation is much simplified by observing that its integral surfaces admit of $U(f)$.

†Cf. LIE-ENGEL, Transformationsgruppen, vol. 2, p. 263.

‡Vide Leipziger Berichte, 1889, p. 153. Cf. also Darboux, Leçons sur les systèmes orthogonaux, vol. 1 , pp. 56, 72, in which $\left(A_{0}\right)$ is called the transformation de Ribaucour. 
where

$$
\begin{gathered}
\gamma=x_{1} p_{2}-x_{2} p_{1}, \quad a=\frac{1}{2} p_{2}\left(u+r_{0}^{2}\right)-x_{2} l, \quad \beta=x_{1} l-\frac{1}{2} p_{1}\left(u+r_{0}^{2}\right), \\
y^{\prime}=\frac{d x_{2}}{d x_{1}}=-\frac{p_{1}}{p_{2}},
\end{gathered}
$$

$$
u=x_{1}^{2}+x_{2}^{2}, \quad l=p_{1} x_{1}+p_{2} x_{2},
$$

we obtain the required equation,

$$
F\left(\frac{1}{2}\left(x^{2}+y^{2}+r_{0}^{2}\right)+y\left(x y^{\prime}-y\right), \frac{y^{\prime}}{2}\left(x^{2}+y^{2}+r_{0}^{2}\right)-x\left(x y^{\prime}-y\right), x+y y^{\prime}\right)=0,
$$

wherein $x$ and $y$ have been written for $x_{1}$ and $x_{2}$, and $F$ is an arbitrary homogeneous function.

The characteristic function for the infinitesimal contact transformation $U(f)$ now takes the form*

$$
W \equiv-\left(x^{2}+y^{2}-r_{0}^{2}\right) \sqrt{1+y^{\prime 2}} .
$$

\section{§6. Some special cases of the preceding results.}

Inversion in a general linear spherical complex is transformed by a spherical inversion whose center lies on the fundamental sphere into inversion in a plane spherical complex, i. e., into the transformation of LAGUerRe. $\dagger$ The fundamental sphere $\sigma$ is replaced by a fundamental plane $\pi$. If $\pi$ be taken for $z=0$, the point-sphere correspondence $\Gamma$ of $\S 1$ becomes

$$
\Phi^{\prime} \equiv\left(1-\kappa_{0}^{2}\right)\left\{(X-x)^{2}+(Y-y)^{2}+(Z-z)^{2}\right\}+4 z Z=0,
$$

and is the most general involutory point-sphere correspondence such that a point $P$ and its associated sphere $S$ have a common radical plane.

The $\infty^{3}$ spheres $S$ belong to a plane complex $E$ with fundamental plane $\pi$ and constant $\kappa=1+\kappa_{0}^{2} / 2 \kappa_{0}$, as before.

Characteristic properties of the transformation are (1), planes transform into planes, the plane at infinity being invariant, and (2), the distance of two points $P$ and $P^{\prime}$ equals the length of a common tangent of the corresponding spheres.

Theorems 1, 2, 3 hold here.

The equations of the contact transformation, i. e., inversion in the complex $E_{0}$ with fundamental plane $z=0$ and constant $\kappa_{0}$ are readily found as before $(\S 2)$. As fundamental configurations of $\left(E_{0}\right)$ appear. $(1)$, the $\infty^{2}$ surface elements whose points lie in $\pi$ and whose planes make with $\pi$ the angle $\cos ^{-1} \kappa$, and (2), the $\infty^{2}$ elements of the imaginary circle at infinity. An element of (1) transforms into the $\infty^{1}$ minimum elements along a minimum line, while to an

\footnotetext{
* Cf. Sophus LIE, Geometrie der Berührungstransformationen, vol. 1, p. 150.
}

† SMrre, Annals of Mathematics, July, 1900. 
element of (2) correspond the $\infty^{2}$ elements of a plane belonging to $E$. Especially noteworthy is the fact that two elements $\left(P_{1}, E\right),\left(P_{2}, E\right)$ having the same plane $E$ go over into elements of the same sort, $\left(P_{1}^{\prime}, E^{\prime}\right),\left(P_{2}^{\prime}, E^{\prime}\right)$ such that $P_{1} P_{2}=P_{1}^{\prime} P_{2}^{\prime}$. This property is expressed thus :

Tangential distance is invariant under inversion in a plane spherical complex.

By a spherical inversion elements having a common point $\left(P, E_{1}\right),\left(P, E_{2}\right)$ transform into elements of like nature $\left(P^{\prime}, E_{1}^{\prime}\right),\left(P^{\prime}, E_{2}^{\prime}\right)$, and such that the angle of $E_{1}$ and $E_{2}$ equals that between $E_{1}^{\prime}$ and $E_{2}^{\prime}$. I have elsewhere bespoken for the transformation of LAAGUERRE an attention it has not as yet received,* and the analogy just remarked would seem to warrant this.

Within the general $G_{15}(\S 3)$ of all sphere-sphere contact transformations in space is contained as subgroup a $G_{10}^{\prime}$ of all such transformations as are compounded of inversions in plane spherical complexes. This $G_{10}^{\prime}$ is the group of the geometry of reciprocal directions. The contact transformation $\left(E_{0}\right)$ inverts the group $G_{6}$ of all transformations compounded of plane reflections into that subgroup $G_{6}^{\prime}$ of $G_{10}^{\prime}$ under which the plane spherical complex $E$ remains invariant. In this way we establish a duality of the Euclidean geometry (excluding similitude) to spherical geometry within a plane spherical complex.

It is not proposed to develop this question. There is of course much in $\S 3$ which is valid here, but we have in addition the very interesting correspondence of distance to tangential distance.

The group of $\infty^{1}$ contact transformations made up of inversions in all plane complexes having the same fundamental plane $x_{3}=0$ is generated by the infinitesimal contact transformation $U(f)$ whose characteristic function is

i. e.,

$$
H \equiv 2 x_{3} s,
$$

$$
U(f)=\frac{2 x_{3}}{s}\left(p_{1} \frac{\partial f}{\partial x_{1}}+p_{2} \frac{\partial f}{\partial x_{2}}+p_{3} \frac{\partial f}{\partial x_{3}}\right)-2 s \frac{\partial f}{\partial p_{3}} .
$$

The general invariant function has the form

$$
F\left(p_{1}, p_{2}, p_{1} x_{1}+p_{2} x_{2}+p_{3} x_{3}, x_{1} p_{2}-x_{2} p_{1}, x_{3} s\right),
$$

$F$ being homogeneous of dimension zero, while every invariant system of $\infty^{4}$ elements is defined by setting any homogeneous function of these arguments equal to zero.

Under the present $G_{\mathrm{I}}$ carried out on the surface element $(P, E)$ the point $P$ describes a circle $c$ whose center lies in the fundamental plane while $E$ turns around the axis $l$ of this circle.

The center of $c$ is

\footnotetext{
* In the Annals of Mathematics, July, 1900.
} 


$$
\left(\frac{p_{1} l+p_{2}\left(x_{1} p_{2}-x_{2} p_{1}\right)}{p_{1}^{2}+p_{2}^{2}}, \frac{p_{2} l-p_{1}\left(x_{1} p_{2}+x_{2} p_{1}\right)}{p_{1}^{2}+p_{2}^{2}}\right),
$$

and its radius equals $x_{3} s / p_{1} p_{2}\left(p_{1}^{2}+p_{2}^{2}\right)^{-1 / 2}$. The axis $l$ intercepts on $O X$ and $O Y$ the distances

$$
\frac{l}{p_{1}}, \frac{i}{p_{2}}
$$

Finally, the preceding discussion put us in a position to solve the problem :

To determine all partial differential equations of the first order whose integral surfaces intersect orthogonally a given system of $\infty^{3}$ circles whose centers lie in a given plane and whose planes are perpendicular to that plane.

If now the fundamental plane of $\Gamma$ is the plane at infinity, the correspondence becomes

$$
(X-x)^{2}+(Y-y)^{2}+(Z-z)^{2}=a^{2} .
$$

The corresponding contact transformation is a dilatation of a point $P$ into the sphere $S$ of constant radius $a$ and center at $P$, while the problem mentioned above becomes this: To determine all partial differential equations of the first order whose integral surfaces have for normals the lines of a given line complex.*

\section{§ 7. Geometrical definition of the correspondence $\Gamma$.}

The subject under discussion appears in a new light from a definition of the fundamental correspondence ( $\$ 1)$ now to be established.

Let $P$ be any point in space $R_{n}$, and $O$ a point in $R_{n+1}$ external to $R_{n}$. Then by a spherical inversion in the center $O$ the minimum cone $V$ of $R_{n+1}$ whose vertex is $P$ transforms into a minimum cone $V^{\prime}$ of vertex $P^{\prime}$. The cone $V^{\prime}$ intersects $R_{n}$ in a sphere $S$, while the sphere $S_{0}$ into which $R_{n}$ inverts cuts $R_{n}$ in a sphere $\sigma$. Also $V$ and $S$ intersect on $\sigma$. Reciprocally, the minimum cone in $R_{n+1}$ with vertex any point $P^{\prime \prime}$ on $S$, passes through $P^{\prime}$, and hence inverts into a minimum cone containing $P$. We see, therefore, that the correspondence of $P$ and $S$ possesses the characteristics of $\Gamma$. This gives

Theorem 7. The point-sphere correspondence $\Gamma$ in $R_{n}$ may be thus defined : $A$ spherical inversion in a center in $R_{n+1}$ external to $R_{n}$ inverts the minimum cone of $R_{n+1}$ whose vertex is a point $P$ of $R_{n}$ into a minimum cone whose intersection with $R_{n}$ is the corresponding sphere $S$.

To proceed to the other properties of $\Gamma$, we choose the sphere as element of $R_{n}$. The $\infty^{n+1}$ points $P^{\prime}$ of $R_{n+1}$ project by minimal projection into the $\infty^{n+1}$ spheres $S$ of $R_{n}$. In fact, the minimum cone in $R_{n+1}$ with vertex $P^{\prime}$ intersects $R_{n}$ in the sphere $S$. We shall lose nothing in generality by confining the discussion to $n=2$; then $R_{2}$ becomes a plane $\pi$; and the $\infty^{3}$ points

* Cf. Sophus Lie, Mathematische Annalen, vol. 5 (1872), p. 204. 
$P^{\prime}$ of space project into the $\infty^{3}$ circles $C$ of $\pi$, such that the center of $C$ is the orthogonal projection of $P^{\prime}$ on $\pi$ while the radius of $C$ equals the ordinate of $P^{\prime}$ relative to $C$ multiplied by $\sqrt{-1}$.*

The $\infty^{2}$ points of any surface $F$ in $R_{3}$ project into the $\infty^{2}$ spheres of $a$ circular complex whose curve of singularities is the intersection with $\pi$ of the minimum developable circumscribed to $F$. In particular, let $F$ be a sphere $S_{0}$. If then $S_{0}$ cuts $\pi$ in the circle $\sigma$ of radius $r_{0}$ and center $O$, a simple calculation shows that any point on $S_{0}$ projects into a circle in $\pi$ of center $c^{\prime}$ and radius $r^{\prime}$ such that

where

$$
\overline{c c^{\prime 2}}-r_{0}^{2}-{r^{\prime}}^{2}-2 r_{0} r^{\prime} \kappa=0,
$$

$$
R_{0}^{2}=r_{0}^{2}\left(1-\dot{\kappa}^{2}\right),
$$

$R_{0}$ being the radius of $S_{0}$. We see then

THEOREM 8. The points of a sphere $S_{0}$ in $R_{n+1}$ project by minimal-projection on $R_{n}$ into the spheres of a linear spherical complex whose fundamental sphere is the intersection of $S_{0}$ and $R_{n}$.

Spherical inversion in the sphere $S_{0}$ in $R_{3}$ goes over into inversion in the corresponding circular complex in $\pi$. Hence the conformal group of point transformations in $R_{3}$ transforms by minimal projection into the group of all circular contact transformations in $\pi$. A plane reflection in $R_{3}$ becomes inversion in a line complex, which, if the plane of the reflexion is parallel to $\pi$, reduces to a dilatation. The theorems proved in $\S \S 2,3$ in the paper already quoted $\dagger$ are readily verified by the known properties of inversions in space.

If now we consider either of the two commutative spherical inversions $\left(I_{0}\right)$ in space by which $\pi$ transforms into the sphere $S_{0}$, then to $\left(I_{0}\right)$ corresponds in $\pi$ by the minimal-projection, the inversion in the circular complex $A_{0}$ to which $\Gamma$ gives rise as a contact transformation. The main results of the previous sections are thus easily established.

Let $C$ be any curve on $S_{0}$, and denote the minimum developable which is the envelope of the minimum cone $V$ whose vertex describes $C$ by $F^{\prime}$. Then $F^{\prime}$ intersects $\pi$ in a curve $c$ which is enveloped by $\infty^{1}$ circles in the circular complex $A$. The locus of centers of the circles enveloping $c$ is the orthogonal projection of $C$ on $\pi$, and $c$ is derived by inversion in a circular complex $A_{0}$ from a primitive curve $c^{\prime}$, regarded as point locus, which is the stereographic projection of $C$ on $\pi$. In particular, if $C$ is a general sphero-quartic, the minimum developable in question envelops a system of confocal cyclides, and is of order 16 passing 8 times through the imaginary

\footnotetext{
* Cf. for further details, LIE, Geometrie der Berülrungstransformationen, vol. 1, p. 437, or KLeIN, Höhere Geometrie, p. 472.

† SMrTh, Transactions, vol. 1 (1900).
} 
circle at infinity and containing five double sphero-quartics of which $C$ is one. The curve $c$ in $\pi$ is therefore the curve of singularities of the general quadratic circular complex, and the properties enumerated in the paper already quoted * follow at once.

We may now state

Theorem 9. Given an $M_{r}$ on a sphere $S_{0}$ in $R_{n+1}$ and let the manifoldness which is the envelope of a minimum cone whose vertex describes $M_{r}$ intersect $R_{n}$ in $M_{n-1}^{\prime}$. Then $M_{n-1}^{\prime}$ is enveloped by $\infty^{r}$ spheres of a linear spherical complex in $R_{n}$, this complex being the locus of the spheres into which the points of $S_{0}$ pass over by minimal projection. The locus of centers of the spheres enveloping $M_{n-1}^{\prime}$ is the orthogonal projection of $M_{r}$ on $R_{n}$, and $M_{n-1}^{\prime}$ may be derived by inversion in a linear spherical complex from a primitive $m_{r}$ regarded as point.locus, viz. the stereographic projection of $M_{r}$ on $R_{n}$.

Sheffield Scientific School, New Haven, Conn., February, 1901.

* Smirh, Transactions, vol. 1, p. 390. 